Revue internationale P.M.E.

Économie et gestion de la petite et moyenne entreprise

Revue

internationale

PME

\title{
Une caractérisation des entreprises manufacturières espagnoles à forte croissance : 1994-1998
}

\author{
José L. Calvo et María José Lorenzo
}

Volume 14, numéro 3-4, 2001

URI : https://id.erudit.org/iderudit/1008697ar

DOI : https://doi.org/10.7202/1008697ar

Aller au sommaire du numéro

Éditeur(s)

Presses de l’Université du Québec

ISSN

0776-5436 (imprimé)

1918-9699 (numérique)

Découvrir la revue

Citer cet article

Calvo, J. L. \& Lorenzo, M. J. (2001). Une caractérisation des entreprises manufacturières espagnoles à forte croissance : 1994-1998. Revue internationale P.M.E., 14(3-4), 45-66. https://doi.org/10.7202/1008697ar
Résumé de l'article

L'article analyse les caractéristiques des petites et moyennes entreprises manufacturières espagnoles à forte croissance (EFC) au cours de la période 1994-1998, à partir d'un échantillon de 1193 entreprises extraites de l'enquête sur les stratégies d'entreprises. Le choix des EFC a été effectué en utilisant un indicateur qui associe la variation absolue et la variation relative de la croissance de l'emploi au cours de cette période. Les résultats amènent à conclure que les PME à forte croissance sont petites et jeunes, et qu'elles appartiennent à des secteurs de haute et moyenne technologie, avec un produit différencié, vendu sur de grands marchés ; elles sont en expansion et ciblent un marché international. En outre, elles innovent, tirent parti des derniers développements technologiques et réalisent des activités de recherche et développement aussi bien directes que complémentaires. De plus, un pourcentage important d'entre elles appartient à un groupe d'entreprises. Leurs caractéristiques indiquent l'existence de traits différentiels par rapport aux autres entreprises appartenant à l'échantillon et surtout par rapport à celles dont l'emploi a diminué durant la période analysée.
Ce document est protégé par la loi sur le droit d'auteur. L'utilisation des services d’Érudit (y compris la reproduction) est assujettie à sa politique d'utilisation que vous pouvez consulter en ligne.

https://apropos.erudit.org/fr/usagers/politique-dutilisation/ 


\title{
Une caractérisation des entreprises manufacturières espagnoles à forte croissance : $1994-1998^{*}$
}

\author{
José L. CALVO \\ María José LORENZO \\ Université nationale d'enseignement à distance de Madrid
}

\section{MOTS CLÉS}

\section{Petites et moyennes entreprises - Croissance de l'emploi Entreprises à forte croissance - Innovation}

\begin{abstract}
RÉSUMÉ
L'article analyse les caractéristiques des petites et moyennes entreprises manufacturières espagnoles à forte croissance (EFC) au cours de la période 1994-1998, à partir d'un échantillon de 1193 entreprises extraites de l'enquête sur les stratégies d'entreprises. Le choix des EFC a été effectué en utilisant un indicateur qui associe
\end{abstract}

\section{LES AUTEURS}

José L. CaLvo détient un doctorat en économie. II a collaboré avec différents organismes en matière de PME et d'innovation technologique (Institut de la petite et moyenne entreprise industrielle; OCDE ; Ministère de la Science et de la Technologie). II a également réalisé plusieurs études dans ces deux secteurs et d'autres en économie industrielle. Actuellement, il est professeur à l'Université nationale d'enseignement à distance (UNED) et collabore avec le Bureau de la science et de la technologie espagnol. Adresse: Facultad de Ciencias Económica y Empresariales, Universidad Nacional de Educación a Distancia, Senda del Rey 11, 28040 Madrid, Espagne. Courriel : <jcalvo@cee.uned.es>.

María JosÉ LoRENZo détient un doctorat en économie et est spécialiste en économie industrielle. Elle a collaboré avec plusieurs institutions relatives aux PME (Institut de la petite et moyenne entreprise ; OCDE) et a publié divers articles sur ces questions. Elle est actuellement professeure à l'Université nationale d'enseignement à distance (UNED) et directrice du Département d'analyse économique I de cette université. Adresse : Facultad de Ciencias Económica y Empresariales, Universidad Nacional de Educación a Distancia, Senda del Rey 11, 28040 Madrid, Espagne. Courriel : <mloren@cee.uned.es>.

* Les auteurs remercient la Fundación Empresa Pública pour leur avoir permis d'utiliser l'enquête sur les stratégies d'entreprises. Ils remercient également les deux correcteurs anonymes pour leurs commentaires et leurs suggestions. 
la variation absolue et la variation relative de la croissance de l'emploi au cours de cette période. Les résultats amènent à conclure que les PME à forte croissance sont petites et jeunes, et qu'elles appartiennent à des secteurs de haute et moyenne technologie, avec un produit différencié, vendu sur de grands marchés; elles sont en expansion et ciblent un marché international. En outre, elles innovent, tirent parti des derniers développements technologiques et réalisent des activités de recherche et développement aussi bien directes que complémentaires. De plus, un pourcentage important d'entre elles appartient à un groupe d'entreprises. Leurs caractéristiques indiquent l'existence de traits différentiels par rapport aux autres entreprises appartenant à l'échantillon et surtout par rapport à celles dont l'emploi a diminué durant la période analysée.

\begin{abstract}
The article analyses the characteristics of Spanish small and medium-sized highgrowth manufacturing companies (EFC) during the 1994-1998 period, based on a sample of 1193 firms taken from the survey on business strategies. The selection of EFCs was made by using an indicator combining absolute and relative employment growth variation in the period. The results suggest that high-growth SMEs are small, young, belonging to medium and high technology sectors, selling their products on wide-spread markets, growing and internationally oriented; they innovate, make use of the latest technological developments and perform both direct and indirect $R-D$ activities. A large number of them also belong to groups of companies. Their characteristics show the existence of features that distinguish them from the other companies included in the sample, and particularly from those with falling employment figures during the period under study.
\end{abstract}

\title{
RESUMEN
}

El artículo analiza las características de las pequeña y medianas empresas manufactureras españolas de alto crecimiento (EFC) durante el período 1994-1998, a partir de una muestra de 1193 empresas extraída de la Encuesta sobre estrategias empresariales. La selección de empresas de las EFC se ha realizado utilizando un indicador que combina la variación absoluta con la variación relativa del crecimiento del empleo en el período. Los resultados sugieren que las PMEs de alto crecimiento son pequeñas, jóvenes, pertenecientes a sectores de tecnología media y alta, con un producto diferenciado, que venden su producto en mercados amplios, en expansión, y con orientación internacional ; que innovan, utilizan los últimos desarrollos tecnológicos, y realizan actividades de I-D tanto directas como complementarias. Un porcentaje importante de ellas, además, pertenece a un grupo de empresas. Sus características permiten suponer la existencia de rasgos diferenciales con respecto a las otras empresas incluidas en la muestra, y especialmente con aquellas que vieron disminuir su empleo durante el período de análisis.

\section{ZUSAMMENFASSUNG}

Der Artikel analysiert die Merkmale der stark wachsenden, kleinen und mittleren Produktionsunternehmen in Spanien während der Periode 1994-1998. Analysiert wurde eine Stichprobe von 1193 ausgewählten Unternehmen in bezug auf die Unternehmensstrategie. 
Die Auswahl der stark wachsenden Unternehmen erfolgte mit einem Indikator, der die absolute Varianz und die relative Varianz des Beschäftigungswachstums während dieser Periode verbindet.

Die Ergebnisse zeigen auf, dass die stark wachsenden KMU klein, jung, zugehörig zu Sektoren mit mittlerer und hoher Technologie, ein differenziertes Produkt haben, und ihre Produkte auf den grossen, wachsenden Märkten mit einer internationalen Ausrichtung verkaufen. Die Unternehmen charakterisieren sich durch ihre Innovationen. Sie wenden die letzten technologischen Entwicklungen an und realisieren direkte und ergänzende Forschungs- und Entwicklungsaktivitäten.

Ein weiterer wichtiger Anteil der Unternehmen gehört zu einer Gruppe, deren Merkmale es erlaubt, die Existenz von unterschiedlichen Charakterzügen aufzuzeigen. Sei es im Vergleich zu den Unternehmen der Stichprobe aber vor allem im Vergleich zu denjenigen Unternehmen, deren Beschäftigungsrate sich während der Beobachtungsperiode verminderte.

\section{Introduction ${ }^{1}$}

L'objectif de ce travail est d'analyser les caractéristiques des petites et moyennes entreprises (PME) manufacturières espagnoles à forte croissance, soit celles qui ont le plus contribué à la création de nouveaux emplois au cours d'une période donnée ${ }^{2}$.

Cette étude a été réalisée en utilisant un échantillon permanent de 1193 entreprises qui, en 1994, avaient moins de 500 employés et dont on possède des informations presque complètes pour l'ensemble de la période analysée, soit 1994-1998. Parmi elles, nous avons retenu comme entreprises à forte croissance celles qui se trouvent dans le percentile 90 de l'indice AGR, indicateur proposé par la Hollande dans l'étude précédente de l'OCDE, et qui permet de sélectionner les entreprises en tenant compte à la fois de la variation absolue et de la variation relative de l'emploi. À partir de cette sélection, nous comparons les caractéristiques des PME à forte croissance (EFC), des entreprises qui ont connu une croissance positive de leur emploi et de celles dont le nombre de travailleurs n'a pas changé ou a diminué au cours de la période de l'étude.

L'analyse des EFC et la comparaison de ces résultats avec le reste des entreprises se font selon une double optique : en premier lieu, une analyse intragroupe, qui observe la distribution interne de l'ensemble de ce type d'entreprises en fonction des groupes définis pour chacune des variables retenues; en second lieu, une étude

1. Une version plus détaillée de cet article apparaît dans Calvo et Lorenzo (2001).

2. Cette dénomination correspond à celle utilisée dans les études menées au sein de l'OCDE sur les high-growth firms (Scherer, 2000).

Revue internationale P.M.E., vol. 14, $\mathrm{n}^{\text {os }} 3-4,2001$ 
entregroupe, qui compare la présence des $\mathrm{EFC}$ dans chaque groupe prédéfini de cette variable. Pour réaliser cette analyse, on intègre ce qu'on appelle la tranche en pourcentage, qui représente la participation des EFC sur un tronçon déterminé de la variable, que ce soit la taille, l'âge, etc.

Quant aux variables qui caractérisent les PME à forte croissance et qui sont analysées dans cet article, leur choix a été fait à partir d'études préalables sur ce type d'entreprises et, particulièrement, en tenant compte de l'imposante littérature qui met en relation la plupart des variables sélectionnées avec la croissance de l'emploi.

Dans la première partie de ce travail, nous faisons référence aux raisons qui justifient le choix des variables qui résument les caractéristiques des entreprises à forte croissance. La deuxième partie présente la méthodologie employée pour choisir ces entreprises et les données utilisées dans l'article. La troisième partie met en relation les deux précédentes et effectue une analyse descriptive des principales caractéristiques des entreprises à forte croissance (EFC) en les comparant à celles observées dans les deux autres sous-échantillons : celles dont l'emploi a augmenté (VEMP $>0$ ) et celles dont le nombre de travailleurs a été maintenu ou a diminué $(\mathrm{VEMP} \leq 0)$. La quatrième partie introduit un test non paramétrique pour déterminer si les caractéristiques analysées permettent d'établir un comportement différentiel entre les entreprises à forte croissance et les autres groupes considérés. Enfin, la cinquième partie résume les principales conclusions de l'étude.

\section{Les caractéristiques des entreprises à forte croissance : choix des variables}

Depuis le travail pionnier de Birch (1979), qui établissait que les PME occupaient une part considérable de l'emploi de l'industrie manufacturière des États-Unis et créaient le plus d'emplois ${ }^{3}$, une large documentation a été développée dans le but de connaître les caractéristiques des entreprises, surtout des PME qui ont enregistré des taux de croissance d'emploi positifs au cours de différentes périodes. Cette section tente de rassembler les résultats les plus significatifs dans ce secteur et de signaler quelles sont les variables généralement utilisées pour mener ce type d'analyse ${ }^{4}$.

3. Ce type de résultats a été obtenu pour différents pays, y compris l'Espagne. Par exemple, dans les rapports annuels de l'Instituto de la Pequeña y Mediana Empresa (IMPI, 1994).

4. Soulignons que dans la plupart des travaux qui analysent le comportement des PME, les variables examinées sont hautement corrélationnelles, c'est pourquoi il est parfois difficile de séparer leurs effets respectifs. Ainsi, par exemple, il existe toute une branche consacrée à l'étude du comportement des entreprises petites, jeunes et innovatrices, aussi bien dans leur processus d'entrée et de sortie des secteurs, que de leur croissance (Almus, Nerlinger et Steil, 1999), dans laquelle, des variables comme la taille, l'âge et le taux 


\subsection{Taille}

Il n'y a pas unanimité chez les experts en ce qui concerne la relation entre la taille des entreprises et la croissance de leur effectif. Mais, malgré la «Loi de Gibrat» (Gibrat, 1931), la plupart des études relèvent l'existence d'une relation négative entre croissance et taille (Evans, 1987 ; Dunne et Hughes, 1994 ; Liu, Tsou et Hammit, 1999; Heshmati, 2001).

Par conséquent, on peut s'attendre à ce que les entreprises à forte croissance soient, en général, de taille réduite. Cette hypothèse doit toutefois être nuancée dans cet article pour plusieurs raisons : en premier lieu, le choix d'entreprises à forte croissance fait à partir de l'indice AGR (que nous verrons par la suite) tente d'éliminer des biais de la taille ; en deuxième lieu, la majorité des travaux empiriques cités précédemment concluent à l'existence d'une relation non linéaire entre la taille et la croissance des entreprises, avec des éléments quadratiques, en association avec l'âge ${ }^{6}$; en troisième lieu, les fondements théoriques de cette relation, liés au concept de taille minimale efficiente, supposent que les entreprises naissent avec une taille inférieure au minimum efficient, et grandissent rapidement jusqu'à atteindre cette taille (Almus et Nerlinger, 2000).

\section{2. Âge}

La seconde hypothèse la plus vérifiée dans les études sur la croissance de l'emploi dans les PME est l'existence d'une relation négative entre l'âge et la croissance de l'emploi : les entreprises les plus jeunes auraient les plus forts taux de croissance (Evans, 1987; Audretsch, 1995 ; Dunne et Hughes, 1994 ; Liu, Tsou et Hammit, 1999 ; Heshmati, 2001; Jovanovic, 2001). On est donc en droit de supposer qu'il existe une relation négative entre l'âge de l'entreprise et sa probabilité d'appartenir au groupe des EFC. Mais, comme pour la taille, les relations observées ne sont généralement pas linéaires.

\subsection{Secteur d'appartenance}

Il existe également de nombreuses études qui analysent la croissance de l'emploi en fonction du degré de développement technologique du secteur dans lesquelles les entreprises exercent leur activité. En ce sens, il faut signaler les études d'Audretsch

de croissance des entreprises sont étudiées (et comparées) dans leur ensemble. Nous analysons, dans ce travail, ces variables séparément afin d'examiner leur influence individuelle sur la croissance des entreprises.

5. Chose qui ne se fait pas dans la plupart des études, où la variable explicative est le taux de croissance de l'emploi.

6. Voir, par exemple, Heshmati (2001).

Revue internationale P.M.E., vol. 14, nºs 3-4, 2001 
(1995), Dunne, Roberts et Samuelson (1989 a et b), Harhoff, Stahl et Woywode (1998) et Almus, Nerlinger et Steil (1999), qui ont relevé des différences intersectorielles considérables sur le plan de la croissance de l'emploi des entreprises ainsi que l'existence d'une relation positive entre le degré de développement du secteur et la croissance.

\subsection{Marchés}

Il existerait une relation entre la dynamique du secteur et la croissance de l'entreprise (Freel, 2000). Concrètement, l'argument est le suivant : la dynamique d'un secteur en expansion engendre des croissances différentes de celles qui dominent dans les secteurs mûrs.

Trois hypothèses ont été faites dans cet article : 1) les EFC travaillent principalement sur de grands marchés, tout en essayant d'atteindre des créneaux de marché dans des zones géographiques proches; 2) les marchés sur lesquels travaillent les EFC sont principalement en phase d'expansion ; 3) la part de marché des EFC est en augmentation.

\subsection{Exportations}

C'est une variable dont l'effet sur la croissance de l'emploi est indéterminé. Ainsi, aussi bien Storey (1994) que Lessat et Woywode (1997) obtiennent des résultats ambigus quant à l'effet de cette variable sur la croissance des entreprises, tandis que des auteurs comme Robson et Bennet (2000), Storey, Watson et Wynarczyk (1989) et Kinsella et al. (1993) concluent leur analyse en confirmant l'existence d'une relation positive entre ces deux variables. Dans le cas espagnol, l'analyse réalisée avec les données de la période 1990-1994 faisait également ressortir une relation positive (Calvo et Lorenzo, 1998).

\subsection{Innovation}

Il existe une importante documentation sur les relations entre la création d'emploi des PME et leur activité innovatrice; habituellement, les entreprises innovatrices (et jeunes) croissent plus rapidement que les autres (Oakey, 1993 ; Licht et Nerlinger, 1998; Storey et Tether, 1998).

Quant à l'innovation de processus ou de produit, son effet sur la création d'emploi peut être ambigu : tandis que l'innovation de produit crée de nouvelles demandes de marché, qui se traduisent par une croissance de l'emploi, les innovations de processus réduisent les coûts et sont généralement accompagnées de réductions d'emploi. C'est pourquoi certaines études empiriques relèvent un impact positif de l'innovation de produit sur l'emploi (Brouwer, Kleinknecht et Reijnen, 
1993 ; Westhead et Cowling, 1995 ; Tether et Massini, 1998 ; Freel, 2000), alors que cette relation n'est pas observée dans les travaux menés par Kalantaridis et Pheby (1999), Wynarczyk et Thwaites (1997), Roper (1997) ou Oakey (1991).

\subsection{Recherche et développement}

Le dernier grand groupe de variables apportées à l'analyse concerne celles liées aux activités de recherche et développement. La majeure partie des études consultées arrivent à établir une relation positive entre R-D (mesurée par différents indicateurs) et croissance de l'emploi. C'est le cas, par exemple, de Brouwer et Kleinknecht (1996) et Freel (2000). La variable utilisée pour cette analyse est la part des dépenses en R-D par rapport aux chiffres d'affaires.

De plus, nous avons inclu deux autres variables à l'analyse : le fait que l'entreprise appartienne ou non à un groupe d'entreprises - l'effet positif de cette variable est vérifié dans les études allemandes et françaises de ce numéro et dans Almus et Nerlinger (2000) - et la différenciation du produit.

\section{Les entreprises à forte croissance : données utilisées et choix des entreprises}

Pour l'analyse menée dans cet article, nous utilisons un échantillon permanent de 1193 entreprises manufacturières espagnoles pérennes au cours de la période 1994-1998. Comptant moins de 500 travailleurs en 1994, elles ont fourni des informations sur les deux périodes (1994 et 1998) sur plusieurs variables examinées dans ce travail. L'échantillon a été créé à partir des informations recueillies dans l'Enquête sur les stratégies d'entreprises (ESEE) menée par la Fundación Empresa Pública.

De cet échantillon permanent et en utilisant l'indice de croissance de l'emploi $\mathrm{AGR}^{7}$, nous avons tiré l'échantillon des entreprises à forte croissance (EFC) en prenant les $10 \%$ qui avaient connu la plus forte croissance de leur force de travail, soit celles se trouvant dans le percentile 90 de la distribution de l'indice.

Plus concrètement, l'indice AGR utilisé pour établir l'échantillon des entreprises à forte croissance $(\mathrm{EFC})^{8}$ est défini comme suit :

$$
\text { AGR }=\left(\frac{\text { emploi98 }- \text { emploi94 }}{\text { emploi94 }}\right)(\text { emploi98 }- \text { emploi94 })^{0,25}
$$

7. Voir l'étude sur les Pays-Bas dans ce numéro.

8. Une analyse détaillée des entreprises à forte croissance, de leurs caractéristiques et du processus de création et destruction d'emploi de l'échantillon sur la période 1990-1994 apparaît dans Calvo et Lorenzo (1998), étude réalisée pour l'OCDE.

Revue internationale P.M.E., vol. 14, $\mathrm{n}^{\text {os }} 3-4,2001$ 
où emploi 98 fait référence au nombre de travailleurs employés par les entreprises en 1998, et emploi94 à l'emploi des mêmes entreprises dans la première année d'information comprise dans l'échantillon (1994). Il s'agit donc d'un indicateur qui combine les variations absolues de l'emploi et le taux de variation, en introduisant une correction dans la première en l'élevant à 0,25 afin d'éliminer les éventuels biais introduits par d'autres indicateurs alternatifs, vers les grandes entreprises (si l'on utilise la variation absolue de l'emploi) ou, au contraire, vers les unités de plus petite taille (si l'on utilise le taux de croissance de l'emploi) ${ }^{9}$.

Conformément au critère de sélection préétabli, le sous-ensemble d'EFC est composé d'entreprises dont l'indice d'AGR est supérieur ou égal à la valeur critique de 1,389, qui délimite le percentile 90 de la distribution.

TABLEAU 1

Caractéristiques de l'indice AGR

\begin{tabular}{lrll}
\hline & AGR & Percentile & AGR \\
\hline Moyenne & 0,57 & Percentile 90 & $\mathbf{1 , 3 8 9}$ \\
Minimum & $-3,27$ & Percentile 95 & 2,34 \\
Maximum & 194,33 & Percentile 99 & 6,84 \\
\hline
\end{tabular}

Source : ESEE98, ESEE94 et nos calculs.

L'importance du rôle joué par les entreprises à forte croissance pour l'emploi est évidente si l'on considère les données présentées au tableau 2 ; on y relève en effet que les 119 entreprises manufacturières qui appartiennent au sous-ensemble des EFC ont été responsables de la création de 12742 nouveaux emplois, ce qui représente $56 \%$ du total des emplois créés au cours de ces quatre années.

TABLEAU 2

\section{Variation de l'emploi entre 1994 et 1998}

\begin{tabular}{lccccc}
\hline & VEMP $>\mathbf{0}$ & VEMP $=\mathbf{0}$ & VEMP $<\mathbf{0}$ & EFC & Total entreprises \\
\hline $\begin{array}{l}\text { Moyenne } \\
\text { Minimum }\end{array}$ & 32,9 & 0 & $-22,9$ & 107,1 & 10,5 \\
Maximum & 1 & 0 & -309 & 6 & -309 \\
\hline Total & 974 & 0 & -1 & 974 & 974 \\
\hline $\begin{array}{l}\text { Nombre } \\
\text { d'observations }\end{array}$ & 22698 & 0 & -10216 & 12742 & 12482 \\
\hline
\end{tabular}

Source : ESE1998; ESEE1994 et nos calculs.

9. Voir Bangma, Van Noort et Verhoeven (1997) pour une analyse détaillée de ces biais. 


\section{Caractéristiques des entreprises à forte croissance}

Dans cette section, nous analyserons certaines des caractéristiques les plus remarquables des EFC, en tâchant de relever celles qu'on ne retrouve pas dans les autres entreprises.

\subsection{La taille de l'entreprise}

La première des caractéristiques analysées est la taille des entreprises, définie dans cette étude par le nombre d'employés de l'entreprise en considérant cinq tranches de taille (tableau 3).

TABLEAU 3

Distribution des entreprises par tranches de taille 1994

\begin{tabular}{lcccccrr}
\hline $\begin{array}{l}\text { TAILLE } \\
\text { (nombre d'employés) }\end{array}$ & \multicolumn{2}{c}{ VEMP $\leq \mathbf{0}$} & \multicolumn{2}{c}{ VEMP $>\mathbf{0}$} & \multicolumn{3}{c}{ EFC } \\
\hline & $\begin{array}{c}\text { Nombre } \\
\text { d'entreprises }\end{array}$ & $\%$ & $\begin{array}{c}\text { Nombre } \\
\text { d'entreprises }\end{array}$ & $\%$ & $\begin{array}{c}\text { Nombre } \\
\text { d'entreprises }\end{array}$ & $\%$ & $\begin{array}{c}\text { Tranche } \\
\%\end{array}$ \\
\hline$\leq 20$ & 150 & 29,8 & 204 & 35,8 & 37 & 31,1 & 9,5 \\
$21-50$ & 120 & 23,8 & 136 & 23,9 & 26 & 21,8 & 9,2 \\
$51-100$ & 34 & 6,7 & 41 & 7,2 & 10 & 8,4 & 11,8 \\
$101-200$ & 59 & 11,7 & 62 & 10,9 & 20 & 16,8 & 14,2 \\
$201-499$ & 141 & 28,0 & 127 & 22,3 & 26 & 21,8 & 8,8 \\
\hline Total & 504 & 100 & 570 & 100 & 119 & 100 & 10,0 \\
\hline
\end{tabular}

Source : ESEE94 et élaboration personnelle.

Comme on le voit au tableau 3, la distribution des entreprises par tranche de taille dans chacun des sous-échantillons a une forme de U. On observe que le pourcentage des entreprises dans chaque tranche décroît en même temps que la taille de l'entreprise, jusqu'à 100 employés. Ce pourcentage connaît une nette augmentation à partir de cette tranche.

Signalons que les PME à forte croissance sont surtout concentrées dans les plus petites entreprises; celles qui ont 50 employés ou moins représentent presque $53 \%$ du total des EFC; de leur côté, celles qui ont plus de 100 travailleurs comptent pour 38,6\% du total et ce sont les entreprises avec 51 à 100 employés qui ont la plus petite participation dans le sous-ensemble des EFC $(8,4 \%)$.

Ainsi, les résultats commentés dans les paragraphes précédents ne semblent pas confirmer l'hypothèse de l'existence d'une relation négative entre la taille des entreprises et la croissance de leur emploi, bien que les EFC se concentrent surtout dans les tranches de taille inférieures.

Revue internationale P.M.E., vol. 14, nos 3-4, 2001 
Toutefois, dans la dernière colonne du tableau $3^{10}$, les valeurs supérieures à $10 \%$ se traduisent par une participation des entreprises à forte croissance supérieure à celle qui leur correspondrait si la distribution était homogène entre toutes les tranches de taille. En revanche, les valeurs inférieures à $10 \%$ reflètent une sous-représentation des EFC dans cette tranche de taille. De plus, au fur et à mesure qu'augmente la valeur comprise dans cette colonne, la probabilité qu'une entreprise d'une tranche déterminée fasse partie du groupe des EFC augmente également.

Par conséquent, conformément à cette interprétation, on détecte l'existence d'un certain biais à la hausse dans la représentation des entreprises qui ont entre 50 et 200 employés et, surtout, les plus grandes parmi celles-ci (entre 101 et 200 employés); en revanche, contrairement aux précédentes, les entreprises dont le nombre d'employés est supérieur à 200 et inférieur à 50 ont une participation inférieure à celle qui leur correspondrait si la distribution de l'échantillon des EFC était uniforme.

\subsection{Ancienneté des entreprises}

En poursuivant notre analyse, le tableau 4 présente la distribution des entreprises selon leur ancienneté.

TABLEAU 4

Distribution des entreprises selon leur ancienneté 1994

\begin{tabular}{lccccccr}
\hline $\begin{array}{l}\text { Ancienneté } \\
\text { (années) }\end{array}$ & \multicolumn{2}{c}{ VEMP $\leq \mathbf{0}$} & \multicolumn{2}{c}{ VEMP $>\mathbf{0}$} & \multicolumn{2}{c}{ EFC(1) } \\
\hline & $\begin{array}{c}\text { Nombre } \\
\text { d'entreprises }\end{array}$ & $\%$ & $\begin{array}{c}\text { Nombre } \\
\text { d'entreprises }\end{array}$ & $\%$ & $\begin{array}{c}\text { Nombre } \\
\text { d'entreprises }\end{array}$ & $\%$ & $\begin{array}{c}\text { Tranche } \\
(\%)\end{array}$ \\
\hline $0-5$ & 36 & 7,1 & 37 & 6,5 & 6 & 5,0 & 7,6 \\
$6-10$ & 62 & 12,3 & 120 & 21,1 & 34 & 28,6 & 15,7 \\
$11-20$ & 133 & 26,4 & 183 & 32,1 & 36 & 30,3 & 10,2 \\
$21-30$ & 88 & 17,5 & 94 & 16,5 & 20 & 16,8 & 9,9 \\
Plus de 30 & 185 & 36,7 & 136 & 23,9 & 23 & 19,3 & 6,7 \\
\hline Total & 504 & 100 & 570 & 100 & 119 & 100 & 10,0 \\
\hline
\end{tabular}

Source : ESEE94 et élaboration personnelle.

L'analyse permet de relever d'importantes différences entre la distribution par âge des EFC et celle du reste des entreprises, particulièrement celles qui connaissent une variation négative de l'emploi. Ainsi, tandis qu'au moins une

10. Concrètement, et pour la première tranche, ce serait $(37 / 391) * 100$. 
entreprise à forte croissance sur trois a été fondée après 1984, presque $40 \%$ de celles qui ont maintenu ou réduit leur nombre d'employés ont plus de 30 ans d'existence. C'est dans le groupe de 6 à 10 ans (entreprises créées à partir de 1984) que l'on observe la plus grande différence, car presque $30 \%$ des EFC sont concentrées dans cette tranche et seulement $12 \%$ de celles de variation négative, ce qui fait que le pourcentage de tranche s'élève à $15,7 \%$. Bref, plus de $60 \%$ des entreprises ayant connu une croissance positive de leur emploi peuvent être qualifiées de jeunes, car elles ont été fondées après 1974.

\subsection{Appartenance à un groupe d'entreprises}

Une autre caractéristique qui peut différencier les EFC du reste des entreprises est leur appartenance ou non à une société ou groupe d'entreprises. Les résultats pour le cas espagnol avec ce nouvel échantillon sont présentés dans le tableau 5.

TABLEAU 5

Distribution des entreprises selon l'appartenance à un groupe d'entreprises 1998

\begin{tabular}{lccccccc}
\hline Appartenance & \multicolumn{2}{c}{ VEMP $\leq \mathbf{0}$} & \multicolumn{2}{c}{ VEMP $>\mathbf{0}$} & \multicolumn{2}{c}{ EFC } \\
\hline & $\begin{array}{c}\text { Nombre } \\
\text { d'entreprises }\end{array}$ & $\%$ & $\begin{array}{c}\text { Nombre } \\
\text { d'entreprises }\end{array}$ & $\%$ & $\begin{array}{c}\text { Nombre } \\
\text { d'entreprises }\end{array}$ & $\begin{array}{c}\text { Tranche } \\
(\boldsymbol{\%})\end{array}$ \\
\hline Appartient & 161 & 32,6 & 150 & 26,5 & 46 & 39,0 & 12,9 \\
N'appartient pas & 333 & 67,4 & 415 & 73,5 & 72 & 61,0 & 8,8 \\
\hline Total & 494 & 100 & 565 & 100 & 118 & 100 & 10,0 \\
\hline
\end{tabular}

Source : ESEE98 et nos calculs.

Presque $40 \%$ des EFC appartiennent à un groupe, pourcentage qui descend à $26 \%$ pour les entreprises ayant crû modérément et à $33 \%$ pour celles dont le nombre d'employés n'a pas changé ou a diminué. On peut donc affirmer qu'il existe un certain effet différentiel. De plus, le fait que le pourcentage des EFC dans les entreprises appartenant à un groupe dépasse $10 \%$ suppose une surreprésentation de ce type d'entreprises parmi celles qui appartiennent à des groupes.

\subsection{Différenciation du produit}

Les EFC ne se distinguent pas vraiment des entreprises qui ont connu une croissance d'emploi en fonction du degré de standardisation du produit qu'elles offrent, mais on note une différence importante avec le sous-échantillon d'entreprises qui ont réduit leur effectif.

Revue internationale P.M.E., vol. 14, $\mathrm{n}^{\text {os }} 3-4,2001$ 
TABLEAU 6

Distribution des entreprises selon le degré de standardisation du produit 1998

\begin{tabular}{lccccccc}
\hline & \multicolumn{2}{c}{ VEMP $\leq \mathbf{0}$} & \multicolumn{2}{c}{ VEMP $>\mathbf{0}$} & \multicolumn{2}{c}{ EFC } & \\
\hline & $\begin{array}{c}\text { Nombre } \\
\text { d'entreprises }\end{array}$ & $\%$ & $\begin{array}{c}\text { Nombre } \\
\text { d'entreprises }\end{array}$ & $\%$ & $\begin{array}{c}\text { Nombre } \\
\text { d'entreprises }\end{array}$ & $\%$ & $\begin{array}{c}\text { Tranche } \\
(\boldsymbol{\%})\end{array}$ \\
\hline $\begin{array}{l}\text { Conception } \\
\text { spéciale pour }\end{array}$ & 135 & 27,5 & 228 & 41,2 & 53 & 46,1 & 12,7 \\
$\begin{array}{l}\text { le client } \\
\text { Très standardisé }\end{array}$ & 356 & 72,5 & 326 & 58,8 & 62 & 53,9 & 8,3 \\
\hline Total & 491 & 100 & 554 & 100 & 115 & 100 & 9,9 \\
\hline
\end{tabular}

Source : ESEE98 et nos calculs.

\subsection{Développement technologique du secteur}

Diverses études ont montré que les entreprises œuvrant dans des secteurs à fort contenu technologique ont plus tendance à grandir que celles des secteurs plus traditionnels. Le tableau 7 présente l'information relative au degré de développement technologique du secteur où les entreprises exerçaient leur activité en 1998; nous avons tenu compte de trois niveaux de développement : haut, moyen et bas ${ }^{11}$.

TABLEAU 7

Distribution des entreprises selon le degré de développement technologique de leur secteur

(1998)

\begin{tabular}{lccccccc}
\hline & \multicolumn{2}{c}{ VEMP $\leq \mathbf{0}$} & \multicolumn{2}{c}{ VEMP $>\mathbf{0}$} & \multicolumn{2}{c}{ EFC } & \\
\hline & $\begin{array}{c}\text { Nombre } \\
\text { d'entreprises }\end{array}$ & $\%$ & $\begin{array}{c}\text { Nombre } \\
\text { d'entreprises }\end{array}$ & $\%$ & $\begin{array}{c}\text { Nombre } \\
\text { d'entreprises }\end{array}$ & $\%$ & $\begin{array}{c}\text { Tranche } \\
(\boldsymbol{\%})\end{array}$ \\
\hline $\begin{array}{l}\text { Haute } \\
\text { technologie }\end{array}$ & 126 & 25,0 & 166 & 29,1 & 39 & 32,8 & 11,8 \\
$\begin{array}{l}\text { Moyenne } \\
\text { technologie }\end{array}$ & 136 & 26,9 & 166 & 29,1 & 39 & 32,8 & 11,4 \\
$\begin{array}{l}\text { Basse } \\
\text { technologie }\end{array}$ & 242 & 48,1 & 238 & 41,8 & 41 & 34,4 & 7,9 \\
\hline Total entreprises & 504 & 100 & 568 & 100 & 119 & 100 & 10,0 \\
\hline
\end{tabular}

Source : ESEE98 et nos calculs.

11. Dans le travail de Calvo et Lorenzo (2001), nous avons détaillé les secteurs inclus dans chaque catégorie. 
Il semble exister une relation positive entre le degré de développement technologique du secteur et la probabilité d'appartenir au groupe des EFC. Les entreprises à forte croissance se concentrent principalement dans les secteurs de haute et moyenne technologie et la présence des EFC dans certains secteurs de haute technologie est très importante et dépasse $25 \%$. L'effet différentiel s'observe fondamentalement sur les entreprises qui ont perdu de l'emploi au cours de la période d'étude : tandis que presque la moitié de celles-ci $(48 \%)$ se concentrent sur des secteurs à faible contenu technologique, seulement $34 \%$ des EFC appartiennent à ces secteurs.

\subsection{Marchés}

Les données du tableau 8 font ressortir les différences qui existent entre les EFC et le reste des entreprises de l'échantillon selon la taille géographique du marché auquel est destiné le produit principal de l'entreprise. On peut voir qu'aussi bien les EFC que le reste des entreprises agissent d'abord sur des marchés nationaux. De plus, on observe que tandis que $31 \%$ des EFC se situent, indistinctement, sur des marchés nationaux et étrangers, dans les deux sous-ensembles d'entreprises restantes, ce taux est dans les deux cas inférieur à $22 \%$.

Cette information semble indiquer que les EFC ont tendance à exercer leurs activités sur des marchés de plus grande étendue géographique. Cette hypothèse est corroborée par les taux de la dernière colonne du tableau 8 où l'on observe que la probabilité d'appartenir au groupe des EFC augmente à mesure que s'étend le marché ciblé par ces entreprises. Ce marché est plus étendu pour les unités qui œuvrent aussi bien sur le plan national qu'international.

\section{TABLEAU 8}

Distribution des entreprises selon l'étendue de leur principal marché en 1998

\begin{tabular}{lccccccc}
\hline & \multicolumn{2}{c}{ VEMP $\leq \mathbf{0}$} & \multicolumn{2}{c}{ VEMP $>\mathbf{0}$} & \multicolumn{2}{c}{ EFC } \\
& $\begin{array}{c}\text { Nombre } \\
\text { d'entreprises }\end{array}$ & $\%$ & $\begin{array}{c}\text { Nombre } \\
\text { d'entreprises }\end{array}$ & $\%$ & $\begin{array}{c}\text { Nombre } \\
\text { d'entreprises }\end{array}$ & $\%$ & $\begin{array}{c}\text { Tranche } \\
(\%)\end{array}$ \\
\hline Local & 38 & 7,5 & 49 & 8,6 & 5 & 4,2 & 5,4 \\
Provincial & 60 & 11,9 & 74 & 13,0 & 7 & 5,9 & 5,0 \\
Régional & 60 & 11,9 & 57 & 10,0 & 15 & 12,6 & 11,4 \\
National & 210 & 41,7 & 227 & 39,8 & 47 & 39,5 & 9,7 \\
Étranger & 28 & 5,6 & 39 & 6,8 & 8 & 6,7 & 10,7 \\
Espagne & 108 & 21,4 & 124 & 21,8 & 37 & 31,1 & 13,8 \\
et étranger & 504 & 100 & 570 & 100 & 119 & 100 & 10,0 \\
\hline Total & & & & & & & \\
\hline
\end{tabular}

Source : ESEE98 et nos calculs. 
De plus, comme on peut le déduire des donnés présentées au tableau 9, les EFC se situent de préférence sur des marchés en expansion; seulement 5 PME à forte croissance sur 119 se trouvent sur des marchés en phase de récession.

TABLEAU 9

Distribution des entreprises suivant le dynamisme de leur principal marché en 1998

\begin{tabular}{lcccrrrr}
\hline & \multicolumn{2}{c}{ VEMP $\leq \mathbf{0}$} & \multicolumn{2}{c}{ VEMP $>\mathbf{0}$} & \multicolumn{2}{c}{ EFC } \\
\hline & $\begin{array}{c}\text { Nombre } \\
\text { d'entreprises }\end{array}$ & $\%$ & $\begin{array}{c}\text { Nombre } \\
\text { d'entreprises }\end{array}$ & $\%$ & $\begin{array}{c}\text { Nombre } \\
\text { d'entreprises }\end{array}$ & $\%$ & $\begin{array}{c}\text { Tranche } \\
(\boldsymbol{\%})\end{array}$ \\
\hline Expansif & 150 & 29,8 & 219 & 38,4 & 64 & 53,8 & 14,8 \\
Stable & 282 & 56,0 & 298 & 52,3 & 50 & 42,0 & 7,9 \\
Récession & 72 & 14,3 & 53 & 9,3 & 5 & 4,2 & 3,8 \\
\hline Total entreprises & 504 & 100 & 570 & 100 & 119 & 100 & 10,0 \\
\hline
\end{tabular}

Source : ESEE98 et nos calculs.

TABLEAU 10

Distribution des entreprises selon l'évolution de leur principal marché en 1998

\begin{tabular}{lccccccc}
\hline & \multicolumn{2}{c}{ VEMP $\leq \mathbf{0}$} & \multicolumn{2}{c}{ VEMP $>\mathbf{0}$} & \multicolumn{2}{c}{ EFC } \\
\hline & $\begin{array}{c}\text { Nombre } \\
\text { d'entreprises }\end{array}$ & $\%$ & $\begin{array}{c}\text { Nombre } \\
\text { d'entreprises }\end{array}$ & $\%$ & $\begin{array}{c}\text { Nombre } \\
\text { d'entreprises }\end{array}$ & $\%$ & $\begin{array}{c}\text { Tranche } \\
(\boldsymbol{\%})\end{array}$ \\
\hline A augmenté & 114 & 22,6 & 209 & 36,7 & 53 & 44,5 & 14,1 \\
Constant & 309 & 61,3 & 315 & 55,3 & 57 & 47,9 & 8,4 \\
A diminué & 81 & 16,1 & 46 & 8,1 & 9 & 7,6 & 6,6 \\
\hline Total entreprises & 504 & 100 & 570 & 100 & 119 & 100 & 10,0 \\
\hline
\end{tabular}

Source : ESEE98 et nos calculs.

Enfin, et en ce qui concerne l'évolution des parts de marché respectives, les données du tableau 10 montrent certaines différences entre les trois groupes d'entreprise considérés. Une fois de plus, la dernière colonne de ce tableau révèle que la probabilité d'appartenir au groupe des EFC est supérieure pour les entreprises dont la part de marché a augmenté pendant l'année de référence (1998).

On peut donc affirmer que les EFC agissent fondamentalement sur des marchés plus larges et que l'évolution, aussi bien de leur marché principal que de leur part sur ce marché, a été expansive. 
La dernière variable prise en compte dans cette analyse des marchés exploités par les entreprises de l'échantillon se rapporte à l'activité d'exportation et d'importation. Dans cette étude, les exportations ont été comprises comme une variable dichotomique prenant la valeur 1 si l'entreprise exporte et 0 dans le cas contraire. Une autre variable dichotomique a été utilisée pour les importations.

TABLEAU 11

Distribution des entreprises suivant leur relation avec les marchés extérieurs

1998

\begin{tabular}{lccccccr}
\hline & \multicolumn{2}{c}{ VEMP $\leq \mathbf{0}$} & \multicolumn{2}{c}{ VEMP $>\mathbf{0}$} & \multicolumn{2}{c}{ EFC } \\
& $\begin{array}{c}\text { Nombre } \\
\text { d'entreprises }\end{array}$ & $\mathbf{\%}$ & $\begin{array}{c}\text { Nombre } \\
\text { d'entreprises }\end{array}$ & $\begin{array}{c}\text { \% } \\
\text { Nombre } \\
\text { d'entreprises }\end{array}$ & $\begin{array}{c}\text { No } \\
\text { Tranche } \\
(\boldsymbol{\%})\end{array}$ \\
\hline A augmenté & 114 & 22,6 & 209 & 36,7 & 53 & 44,5 & 14,1 \\
Exportent & 299 & 59,3 & 353 & 6,9 & 91 & 76,5 & 12,2 \\
N'exportent pas & 205 & 40,7 & 217 & 38,1 & 28 & 23,5 & 6,2 \\
Importent & 293 & 58,1 & 335 & 58,8 & 88 & 74,6 & 12,3 \\
N'importent pas & 211 & 41,9 & 235 & 41,2 & 30 & 25,4 & 6,3 \\
\hline Total entreprises & 503 & 100 & 568 & 100 & 119 & 100 & 10,0 \\
\hline
\end{tabular}

Source : ESEE98 et nos calculs.

Le pourcentage des entreprises dont l'activité est orientée vers les marchés extérieurs, qu'il s'agisse d'exportations ou d'importations, est très supérieur dans le groupe des EFC à celui que l'on retrouve dans les entreprises des deux autres sous-échantillons.

\subsection{Innovation}

Nous avons résumé dans le tableau 12 l'information disponible sur l'activité innovatrice des entreprises en différenciant innovation de processus, innovation de produit et innovation de processus ou de produit ${ }^{12}$.

Comme on peut le voir dans ce tableau, pour chacun des sous-échantillons considérés, le pourcentage d'entreprises innovatrices est supérieur dans les EFC à celui observé dans les autres entreprises, peu importe si celles-ci ont connu une réduction ou une augmentation d'emploi.

12. Dans l'étude de Calvo et Lorenzo (2001), nous tenons compte aussi du type de technologie employée par l'entreprise et, concrètement, du fait qu'elle tire parti ou non des dernières découvertes technologiques : machines-outils à contrôle numérique, robotique, etc. Les effets de ces variables sur la croissance sont positifs. 
TABLEAU 12

Distribution des entreprises en fonction de l'introduction d'innovations de processus ou de produit

1998

\begin{tabular}{|c|c|c|c|c|c|c|c|}
\hline & \multicolumn{2}{|c|}{ VEMP $\leq \mathbf{0}$} & \multicolumn{2}{|c|}{ VEMP $>0$} & \multicolumn{2}{|l|}{ EFC } & \multirow[b]{2}{*}{$\begin{array}{c}\text { Tranche } \\
(\%)\end{array}$} \\
\hline & $\begin{array}{c}\text { Nombre } \\
\text { d'entreprises }\end{array}$ & $\%$ & $\begin{array}{c}\text { Nombre } \\
\text { d'entreprises }\end{array}$ & $\%$ & $\begin{array}{c}\text { Nombre } \\
\text { d'entreprises }\end{array}$ & $\%$ & \\
\hline \multicolumn{8}{|c|}{ Innovations de produit } \\
\hline Introduit & 129 & 25,6 & 149 & 26,1 & 38 & 31,9 & 12,0 \\
\hline N'introduit pas & 375 & 74,4 & 421 & 73,9 & 81 & 68,1 & 9,2 \\
\hline \multicolumn{8}{|c|}{ Innovations de processus } \\
\hline Introduit & 148 & 29,4 & 225 & 39,5 & 54 & 45,4 & 10,2 \\
\hline N'introduit pas & 356 & 70,6 & 345 & 60,5 & 65 & 54,6 & 8,5 \\
\hline \multicolumn{8}{|c|}{ Innovations de processus ou de produit (entreprises innovatrices) } \\
\hline Introduit & 213 & 42,3 & 280 & 49,1 & 68 & 57,1 & 12,1 \\
\hline N'introduit pas & 291 & 57,7 & 290 & 50,9 & 51 & 42,9 & 8,0 \\
\hline Total entreprises & 504 & 100 & 570 & 100 & 119 & 100 & 10,0 \\
\hline
\end{tabular}

Source : ESEE98 et nos calculs.

Les deux dernières lignes du tableau 12 montrent la distribution des entreprises qui introduisent un type d'innovation, que ce soit de processus ou de produit ${ }^{13}$ (ce qui habituellement définit une entreprise innovatrice) et celles qui n'en introduisent pas. Les différences, dans ce cas, augmentent ${ }^{14}$.

\subsection{Activités de recherche et développement}

Le dernier aspect de cette analyse différentielle des entreprises manufacturières espagnoles à forte croissance est l'activité de recherche et développement. Comme nous l'avons déjà signalé dans la première section, la grande majorité des études trouvent une relation positive entre ce type d'activités et la croissance de l'emploi des entreprises.

13. Cela signifie qu'une entreprise est sélectionnée si elle a introduit des innovations de produit ou de processus, ou les deux.

14. Il convient de signaler que le pourcentage d'entreprises innovatrices tiré de l'ESEE est excessivement élevé. Pour 1998, le pourcentage des entreprises manufacturières espagnoles était seulement de $10 \%$ (INE, 1999).

Revue internationale P.M.E., vol. 14, $\mathrm{n}^{\text {os }} 3-4,2001$ 
TABLEAU 13

Distribution des entreprises en fonction du taux des dépenses en R-D par rapport aux ventes

1998

\begin{tabular}{lccccccc}
\hline & \multicolumn{2}{c}{ VEMP $\leq \mathbf{0}$} & \multicolumn{2}{c}{ VEMP $>\mathbf{0}$} & \multicolumn{2}{c}{ EFC } \\
\hline $\begin{array}{l}\text { Taux de } \\
\text { dépenses } \\
\text { en R-D }\end{array}$ & $\begin{array}{c}\text { Nombre } \\
\text { d'entreprises }\end{array}$ & $\%$ & $\begin{array}{c}\text { Nombre } \\
\text { d'entreprises }\end{array}$ & $\%$ & $\begin{array}{c}\text { Nombre } \\
\text { d'entreprises }\end{array}$ & $\%$ & $\begin{array}{c}\text { Tranche } \\
(\%)\end{array}$ \\
\hline $0,1-1 \%$ & & & & & & & \\
$1,1-4 \%$ & 90 & 17,9 & 110 & 19,4 & 28 & 23,9 & 12,3 \\
$4,1-6 \%$ & 59 & 11,7 & 75 & 13,2 & 19 & 16,2 & 12,4 \\
Plus de 6\% & 7 & 1,4 & 7 & 1,2 & 4 & 3,4 & 22,2 \\
\hline Total & 9 & 1,8 & 15 & 2,6 & 0 & 0 & 0 \\
entreprises & 503 & 100 & 568 & 100 & 118 & 100 & 9,8 \\
\hline
\end{tabular}

Source : ESEE98 et nos calculs.

Les données du tableau 13 confirment l'existence d'une relation positive entre l'effort de recherche par les entreprises et la probabilité d'appartenir au groupe de celles qui ont le plus grand indice AGR, à l'exception de la dernière tranche de ce tableau, c'est-à-dire celle qui correspond aux entreprises qui consacrent plus de $6 \%$ de leurs ventes à des activités de R-D.

\section{Test non paramétrique d'indépendance}

Cette section présente les résultats obtenus pour le test de Kruskal-Wallis, test non paramétrique d'indépendance des distributions des trois sous-échantillons utilisés au cours de l'analyse ${ }^{15}$. À l'aide de ce test (qui présente les valeurs d'une distribution $\chi^{2}$ avec $n-1$ degré de liberté, $n$ étant le nombre d'échantillons indépendants), il est possible de vérifier si une variable donnée implique un comportement différent dans les trois groupes étudiés et, plus précisément, si cette variable peut servir à différencier les PME à forte croissance de celles dont l'emploi a augmenté et de celles qui n’ont pas modifié le nombre des salariés ou en ont licencié.

15. De fait, les tests non paramétriques pour plusieurs échantillons indépendants « $[\ldots]$ comparent deux groupes de cas ou plus par rapport à une variable en faisant ressortir son homogénéité, c'est-à-dire s'ils proviennent de la même population [...] », le test $\mathrm{H}$ de Kruskal-Wallis étant « [...] une extension du test U de Mann-Whitney, équivalent non paramétrique de l'analyse de variance d'un facteur qui détecte les écarts dans la localisation des distributions [...]». (Pérez, 2000) 
Comme nous pouvons l'observer au tableau 14, l'âge de l'entreprise, l'appartenance à un groupe de sociétés, le degré de normalisation du produit, les variables associées à leurs marchés, les innovations de processus, le type de technologie associée (à l'exception du système informatique centralisé) et certaines des activités complémentaires de R-D, en particulier le recrutement de personnel pour ce type d'activités, sont des variables pour lesquelles on relève un comportement différentiel des PME à forte croissance par rapport aux autres sous-échantillons d'entreprises. Les autres variables ne sont pas significatives. La variable taille mérite une mention particulière, vu que la valeur $\chi^{2}$ qui en résulte est plutôt faible, n'étant pas significative à $99 \%$.

TABleAu 14

Test de Kruskal-Wallis d'indépendance des variables (deux degrés de liberté)

\begin{tabular}{|c|c|c|}
\hline Variables & $\chi^{2}$ & $\begin{array}{c}\text { Degré } \\
\text { de signification }\end{array}$ \\
\hline Taille & 5,255 & 0,072 \\
\hline Âge & 27,737 & 0,000 \\
\hline Appartenance à un groupe d'entreprises & 9,918 & 0,007 \\
\hline Différenciation du produit & 26,897 & 0,000 \\
\hline Développement technologique du secteur & 5,935 & 0,051 \\
\hline \multicolumn{3}{|l|}{ Marchés } \\
\hline Étendue du marché principal & 7,984 & 0,018 \\
\hline Dynamisme du marché principal & 31,258 & 0,000 \\
\hline Évolution de la part du marché & 43,154 & 0,000 \\
\hline Exportation & 12,093 & 0,002 \\
\hline Importation & 11,530 & 0,003 \\
\hline \multicolumn{3}{|l|}{ Innovation } \\
\hline Innovations de processus & 17,165 & 0,000 \\
\hline Innovations du produit & 2,052 & 0,358 \\
\hline Innovation de processus ou de produit & 10,478 & 0,005 \\
\hline Machines-outils à contrôle numérique & 10,700 & 0,005 \\
\hline Robotique & 11,719 & 0,003 \\
\hline Conception assistée par ordinateur & 38,611 & 0,000 \\
\hline Système informatique centralisé & 1,163 & 0,559 \\
\hline Nouvelles technologies & 24,055 & 0,000 \\
\hline \multicolumn{3}{|l|}{ Recherche et développement (R-D) } \\
\hline Activités de R-D & 5,924 & 0,052 \\
\hline Frais en R-D par rapport aux ventes & 4,854 & 0,088 \\
\hline \multicolumn{3}{|l|}{ Activités complémentaires } \\
\hline de recherche et développement & 2,514 & 0,284 \\
\hline Contrôle de recherche et développement & 1,802 & 0,406 \\
\hline Collaboration en innovation & 7,761 & 0,021 \\
\hline Coentreprises & 1,320 & 0,517 \\
\hline \multicolumn{3}{|l|}{ Recrutement de personnel } \\
\hline de recherche et développement & 37,694 & 0,000 \\
\hline Évaluation du changement technique & 8,624 & 0,013 \\
\hline Activités complémentaires & 21,561 & 0,000 \\
\hline
\end{tabular}




\section{Conclusion}

Tout au long de cette étude, nous avons analysé les caractéristiques d'un échantillon de PME à forte croissance (EFC) de l'industrie manufacturière espagnole pour la période 1994-1998, en comparant ces données avec celles des entreprises qui ont connu une augmentation d'emploi et avec celles qui ont connu une stagnation d'emploi ou ont procédé à des licenciements. Les principaux résultats de cette analyse sont les suivants :

- les PME à forte croissance sont principalement des petites entreprises, puisque plus de la moitié d'entre elles emploient moins de 50 personnes. Mais la différence n'est pas significative. D'ailleurs, les données semblent indiquer l'existence d'un certain biais dans l'ensemble des EFC, tendant à accorder une plus grande représentation aux entreprises de plus grande taille;

- les EFC sont des entreprises jeunes, puisqu'une sur trois a moins de dix ans et les deux tiers ont été fondées à partir de 1974. En outre, plus de $60 \%$ des entreprises qui ont connu une croissance positive de l'emploi ont moins de vingt ans ;

- $40 \%$ des PME à forte croissance appartiennent à un groupe d'entreprises ; cette variable est significative par rapport aux autres groupes de l'échantillon;

- plus de $40 \%$ des entreprises qui ont connu une croissance positive de l'emploi produisent des biens conçus spécialement pour le client. Ce pourcentage s'élève à presque la moitié des entreprises à forte croissance. Au contraire, les trois quarts des entreprises dont le personnel a diminué produisent des biens standardisés ;

- les entreprises avec des variations positives de l'emploi se concentrent dans les secteurs de haute et moyenne technologie, tandis que presque la moitié des entreprises qui ont licencié appartiennent à des secteurs à faible contenu technologique. La présence des EFC est particulièrement importante dans les secteurs de l'automobile, de l'informatique et de la bureautique, alors qu'elle est presque symbolique dans d'autres comme ceux des boissons, du cuir, de la fourrure et de la chaussure;

- les PME à forte croissance vendent leurs produits sur de larges marchés, en expansion; elles ont augmenté leur part de marché en 1998. Elles se différencient du reste des entreprises et particulièrement de celles dont l'emploi a diminué. De plus, presque les trois quarts sont exportatrices et importatrices ;

Revue internationale P.M.E., vol. 14, nos 3-4, 2001 
- l'introduction d'innovations de processus est également un fait différentiel des PME à forte croissance, de même que l'utilisation des nouvelles technologies dans les processus de production : plus de $80 \%$ tirent profit des plus récentes découvertes technologiques, comparé à environ $60 \%$ pour les autres entreprises;

- les EFC investissent davantage dans les activités de R-D que le reste des entreprises; et le pourcentage des entreprises qui réalisent des activités complémentaires de recherche et développement est également supérieur.

En résumé, les PME à forte croissance sont petites et jeunes et elles appartiennent à des secteurs de haute et moyenne technologie, avec un produit différencié, vendu sur de grands marchés; elles sont en expansion et ciblent un marché international. En outre, il s'agit d'entreprises qui innovent, tirent parti des derniers développements technologiques et réalisent des activités de recherche et développement aussi bien directes que complémentaires. Un pourcentage important d'entre elles appartiennent à un groupe d'entreprises. Leurs caractéristiques permettent d'affirmer l'existence de traits différentiels par rapport aux autres entreprises de l'échantillon et particulièrement par rapport à celles dont l'emploi a diminué durant la période analysée.

\section{Bibliographie}

Almus, M., E. NERLINGER et F. STEIL (1999), « Growth determinants of start-ups in Eastern Germany : a comparison between innovative and non-innovative firms », dans New Technology Based Firms in the 1990s : vol. 4, Londres, Oakey, During et Mukhtar.

Almus, M. et E. NERLINGER (2000), «Testing « Gibrat's Law» for young firms. Empirical results for West Germany », Small Business Economics, vol. 15, p. 1-12.

AudrestCH, D. (1995), Innovation and Industry Evolution, Cambridge, MIT Press.

Bangma, K., E. VAn NoORT et W.H.J. Verhoeven (1997), «Creation and loss of jobs in the Netherlands », Mimeo, Document préparé pour la réunion informelle des experts de l'OCDE à Paris du 30 septembre 1997.

BIRCH, D. (1979), The Job Generation Process, MIT Program on Neighbourhood and Regional Change, Cambridge.

BROUWER, E., A. KLEINKNECHT et J. REIJNEN (1993), « Employment growth and innovation at the firm level », Journal of Evolutionary Economics, vol. 3, p. 153-159.

BRouwer, E. et A. KLEINKNECHT (1996), «Determinants of innovation : a microeconometric analysis of three alternative innovation output indicators », dans A.H. Kleinknetch (dir.), Determinants of Innovation: The Message from New Indicators, Londres, Macmillan, p. 99-124.

CALVO, J.L. (2000), « La innovación tecnológica en los sectores manufactureros Españoles », Departamento de análisis económico, Documento de Trabajo 0001, UNED.

Revue internationale P.M.E., vol. 14, $\mathrm{n}^{\text {os }} 3-4,2001$ 
CAlvo, J.L. et M.J. LoREnZo (1998), «Las empresas manufactureras Españolas de alto crecimiento en el período 1990-1994 », Mimeo, Document préparé pour la réunion informelle des experts de l'OCDE à Paris du 30 septembre 1997.

CALvo, J.L. et M.J. LoRENZo (2001), «Las empresas manufactureras Españolas de alto crecimiento en el período 1994-1998 », Mimeo, UNED, Departamento de Análisis Económico I.

DunNe, P. et A. Hughes (1994), «Age, size, growth and survival : U.K. companies in the 1980's », The Journal of Industrial Economics, vol. 42, p. 115-140.

DunNe, T., M. RoberTS et L. SAMUELSON (1989a), « Patterns of firm entry and exist in the U.S. manufacturing industries », Rand Journal of Economics, vol. 19, p. 495-515.

Dunne, T., M. Roberts et L. SAmuelson (1989b), « The growth and failure of U.S. manufacturing plants », Quarterly Journal of Economics, vol. 104, p. 671-698.

Evans, D. (1987), «Test of alternative theories of firm growth», Journal of Political Economy, vol. 95, p. 657-674.

FREEL, M. (2000), «Do small innovating firms outperform non-innovators? », Small Business Economics, vol. 14, p. 195-210.

GIBRAT, R. (1931), Les inégalités économiques; applications aux inégalités des richesses, à la concentration des entreprises, aux populations des villes, aux statistiques des familles, etc., d' une loi nouvelle, la Loi de l' effet proportionnel, Paris, Librairie du Recueil Sirey.

HARHOFF, D., K. STAHL et M. Woywode (1998), «Legal form, growth and exit of WestGerman firms : empirical results for manufacturing, construction, trade and service industries », Journal of Industrial Economics, vol. 46, p. 453-488.

Heshmati, A. (2001), «On the growth of micro and small firms : evidence from Sweden », Small Business Economics, vol. 17, p. 213-228.

IMPI (1994), Informe Anual de la Pequeña y Mediana Empresa 1993, Instituto de la Pequeña y Mediana Empresa, Madrid.

INE (1999), Encuesta sobre Innovación Tecnológica en las Empresas 1998, Instituto Nacional de Estadística, Madrid.

Jovanovic, B. (2001), «New technology and the small firm », Small Business Economics, vol. 16, p. 53-55.

KAlAntARIDIS, C. et J. PHEBY (1999), « Processes of innovation among manufacturing SMEs : the experience of Bedfordshire », Entrepreneurship and Regional Development, vol. $11, \mathrm{n}^{\mathrm{o}} 1$, p. $57-78$.

Kinsella, R., W. Clarke, D. Coyne, D. Mulvenna et D. Storey (1993), Fast Growth Firms and Selectivity, Dublin, Irish Management Institute.

LESSAT, V. et M. WoYwOdE (1997), « High-growth SME's and employment : assessment of best practise policies », Mimeo, Document préparé pour la réunion informelle des experts de l'OCDE à Paris du 30 septembre 1997.

LICHT, G. et E. NERLINGER (1998), «New technology-based firms in Germany : a survey of the recent evidence », Research Policy, vol. 26, p. 1005-1022.

Revue internationale P.M.E., vol. 14, $\mathrm{n}^{\text {os }} 3-4,2001$ 
LiU, J., M. Tsou et J. Hammit (1999), « Do small plants grow faster? Evidence from Taiwan electronics industry », Economic Letters, vol. 65, p. 121-129.

OAKEY, R. (1991), «High technology small firms : their potential for rapid industrial growth », International Small Business Journal, vol. 9, nº 4, p. 30-42.

OAKEY, R. (1993), «High technology small firms : a more realistic evaluation of their growth potential », dans C. Karlsson, B. Johannisson et J. Storey (dir.), Small Business Dynamics : International, National, and Regional Perspectives, Londres, Routledge, p. 224-241.

PÉreZ, C. (2000), Técnicas Estadísticas con SPSS, Madrid, Prentice Hall.

ROBSON, P. et R. BENNET (2000), «SME growth: the relationship with business advice and external collaboration », Small Business Economics, vol. 15, p. 193-208.

ROPER, S. (1997), «Product innovation and small business growth : a comparison of the strategies of German, UK and Irish companies », Small Business Economics, vol. 9, p. 523-537.

SCHERER, S. (2000), High-Growth Firms and Employment, Cahier de recherche de STI, 2000/3. OCDE.

StOREy, D., R. WATSON et P. WyNARCZYK (1989), Fast Growth Small Business : Case Studies of 40 Small Firms in Northern England, Department of Employment, Research Paper no 67.

STOREY, D. (1994), Understanding the Small Business Sector, Londres, Routledge.

STOREy, D. (1996), The Ten Percenters, Fast Growing SMEs in Great Britain, Deloitte $\&$ Touche Tohmatsu International.

STOREY, D. et B. TheTER (1998), « New technology-based firms in the European Union : an introduction », Research Policy, vol. 26, p. 933-1026.

SutTon, J. (1997), « Gibrat's legacy », Journal of Economic Literature, vol. 35, p. 40-59.

TETHER, B. et S. MASSINI (1998), «Employment creation in small technological and design innovators in the UK during the 1980s », Small Business Economics, vol. 11, $\mathrm{n}^{\circ}$ 4, p. 353-370.

Westhead, P. et M. Cowling (1995), «Employment change in independent ownermanaged high technology firms in Great Britain », Small Business Economics, vol. 7, no 2, p. 111-140.

WYNARCZYK, P. et A. THWAITES (1997), « The economic performance, survival and nonsurvival of innovative small firms », dans R. Oakey et S. Mukhtar (dir.), New Technology Based Firms in the 1990s: Volume III, Londres. 